\title{
PESQUISA EXPLORATÓRIA COM INDICADORES DE SAÚDE ASSOCIADOS AO ATENDIMENTO POR ESGOTAMENTO SANITÁRIO NA ÁREA METROPOLITANA DE BRASÍLIA
}

\section{EXPLORATORY RESEARCH WITH HEALTH INDICATORS ASSOCIATED WITH SEWAGE CARE IN THE METROPOLITAN AREA OF BRASÍLIA}

\author{
Larissa Damascena da Silva ${ }^{(1)}$ \\ Engenheira Ambiental formada pela Universidade de Brasília. Foi membra do Grupo de Extensão em Saneamento \\ Ambiental e Recursos Hídricos do Departamento de Engenharia Civil e Ambiental, Faculdade de Tecnologia, \\ Universidade de Brasília, durante os anos de 2018 e 2019. \\ Ricardo Tezini Minoti(2) \\ Biólogo, Professor do Departamento de Engenharia Civil e Ambiental da Universidade de Brasília.
}

E-mail ${ }^{(1)}$ : damascenalarissa@gmail.com

\section{RESUMO}

O objetivo deste estudo consiste em analisar espacialmente a variabilidade dos indicadores de saúde associados ao atendimento por saneamento básico na Área Metropolitana de Brasília (AMB) por meio de geoprocessamento, no período de 2007 a 2019. Utilizou em sua metodologia a análise espaço-temporal a partir de operações e ferramentas de um Sistema de Informações Geográficas para organizar, cruzar e sobrepor os dados de saneamento do SNIS e dados de saúde do SINAN para compreender a relação entre saúde e saneamento na região. A partir dos resultados das análises da associação de dengue e leptospirose com a cobertura de saneamento, não foi possível verificar se de fato a relação saúde-saneamento se estabelece nessa região. No entanto, o estudo evidencia as diferenças nos indicadores de saneamento na região e a necessidade de diminuição das desigualdades visando à melhoria das condições de saúde da população.

\begin{abstract}
The objective of this study is to spatially analyze the variability of health indicators associated with basic sanitation services in the Metropolitan Area of Brasília (MAB) through geoprocessing, from 2007 to 2019. It used spatiotemporal analysis in its methodology. from the operations and tools of a Geographic Information System to organize, crossreference, and overlay SNIS sanitation data and SINAN health data to understand the relationship between health and sanitation in the region. Based on the results of the analyzes of the association of dengue and leptospirosis with sanitation coverage, it was not possible to verify whether the health-sanitation relationship is established in this region. However, the study highlights the differences in sanitation indicators in the region and the need to reduce inequalities to improve the population's health conditions.
\end{abstract}

Palavras-chave: Saneamento básico. Dengue. Leptospirose. Área Metropolitana de Brasília. Geoprocessamento.

Key words: Sanitation. Dengue. Leptospirosis. Metropolitan Area of Brasília. Geoprocessing.

\section{INTRODUÇÃO}

O acesso à água potável e ao saneamento básico foram reconhecidos como direito humano pela Organização das Nações Unidas (ONU), a qual declara que "o direito a uma água potável, limpa, de qualidade e às instalações sanitárias é um direito humano, indispensável para gozar plenamente do direito à vida" (ONU, 2010).

A Área Metropolitana de Brasília (AMB) é uma das mais importantes regiões de desenvolvimento brasileiras, abrangendo o Distrito Federal (DF) e mais 12 municípios de Goiás. Com exceção do Distrito Federal, os municípios da região possuem realidades muito parecidas, com elevados déficits nos serviços de saneamento, resultantes da ocupação desordenada e da falta de investimentos nesse 
setor.

Em função das diferenças sociais, marcadamente espaciais, Schvarsberg (2017) apresenta a demanda existente aos pesquisadores, gestores públicos em diferentes níveis e aos diversos atores e segmentos sociais, relacionada à compreensão das lógicas que contribuíram para um processo de metropolização desigual e à necessidade de reversão da situação, com a adoção de estratégias que levem ao surgimento de uma AMB socialmente mais justa e ambientalmente menos desequilibrada.

Como forma de ampliar o entendimento sobre a situação atual, as diferenças existentes entre o Distrito Federal e os municípios que compõem a AMB, surge a necessidade de investigação da relação entre a condição de saneamento básico e os impactos na saúde das populações. Ressalta-se que parte dessas comunidades estão associadas aos assentamentos precários ou caracterizam-se como populações em situação de vulnerabilidade.

O presente trabalho foi estruturado como um estudo de caráter exploratório para o entendimento dessa relação, de sua distribuição no espaço da Área Metropolitana de Brasília (DF/GO), tendo como foco o atendimento por esgotamento sanitário e a taxa de ocorrência de Dengue e Leptospirose nas populações. Apesar de as doenças apresentarem sintomas semelhantes, os ciclos epidemiológicos de transmissão são extremamente diferenciados. A Dengue é caracterizada como uma arbovirose, enquanto a Leptospirose é causada por uma bactéria. $\mathrm{O}$ contato direto com a urina de animais infectados ou a exposição à água contaminada são as principais causas. As duas doenças podem causar surtos durante as estações chuvosas e podem ter como fatores agravantes a precariedade ou ausência de saneamento básico.

O objetivo deste trabalho foi analisar, de maneira exploratória, a variabilidade espacial dos indicadores de saúde (Dengue e Leptospirose) quando comparados com os dados de atendimento por esgotamento sanitário na Área Metropolitana de Brasília por meio de geoprocessamento. A análise visou gerar subsídios para um melhor entendimento sobre a precariedade do saneamento e as diferentes realidades existentes em toda a área contemplada pela AMB.

\section{METODOLOGIA}

O trabalho foi desenvolvido por meio de uma sequência de etapas principais. Após a revisão bibliográfica e a definição dos objetivos, houve a definição da área de estudo. Em seguida foram realizados o levantamento e a análise de dados secundários e a espacialização dos dados, no intuito de averiguar o acesso ao saneamento básico e a ocorrência das doenças na AMB (Figura 1).

A região caracteriza-se por apresentar realidades heterogêneas. Apresenta uma diversidade relacionada à falta de infraestrutura urbana devido à ocupação desordenada do entorno imediato do Distrito Federal, contendo regiões que não possuem sistema de abastecimento de água potável, sistemas de esgotamento sanitário e necessitam de desenvolvimento no sistema de manejo de resíduos sólidos, uma região que carece de investimento e planejamento nesse setor (FIGUEIREDO et al., 2017). Essas regiões ocorrem, também, no interior do DF.

Para análise e seleção de indicadores foram utilizadas bases públicas de dados. Para o saneamento básico a fonte de dados foi o Sistema Nacional de Informações sobre Saneamento (SNIS), responsável por indicadores operacionais, socioeconômicos e ambientais dos municípios e seus prestadores de serviço; a fonte para os dados epidemiológicos foi o Sistema de Informação de Agravos de Notificação (SINAN).

A variável relacionada ao saneamento selecionada para a realização do estudo foi o Atendimento Total de Esgoto, caracterizado como o percentual da população urbana e rural atendida com esgotamento sanitário. O período selecionado para a obtenção dos dados foi de 2007 até o ano 2019, escolhido a partir da disponibilidade dos dados nas bases de dados. 
Para a análise espacial dos dados, foi utilizado o software ArcGIS 10.6.1 (ESRI). A licença do software foi disponibilizada pela Universidade de Brasília. A base cartográfica utilizada no trabalho foi a disponibilizada pelo Instituto Brasileiro de Geografia e Estatística (IBGE).

Foram utilizados dois planos de informação distintos, formato shapefile, para cada ano da análise, um contendo os dados de saneamento e outro os dados de doenças. Os dados em forma de planilha de cada ano foram adicionados às feições do arquivo vetorial (shapefile). Os métodos de análises espaciais aplicados neste estudo são os categorizados em formatos de visualização e análise exploratória de dados.

Figura 1 - Malha Municipal da Área Metropolitana de Brasília.

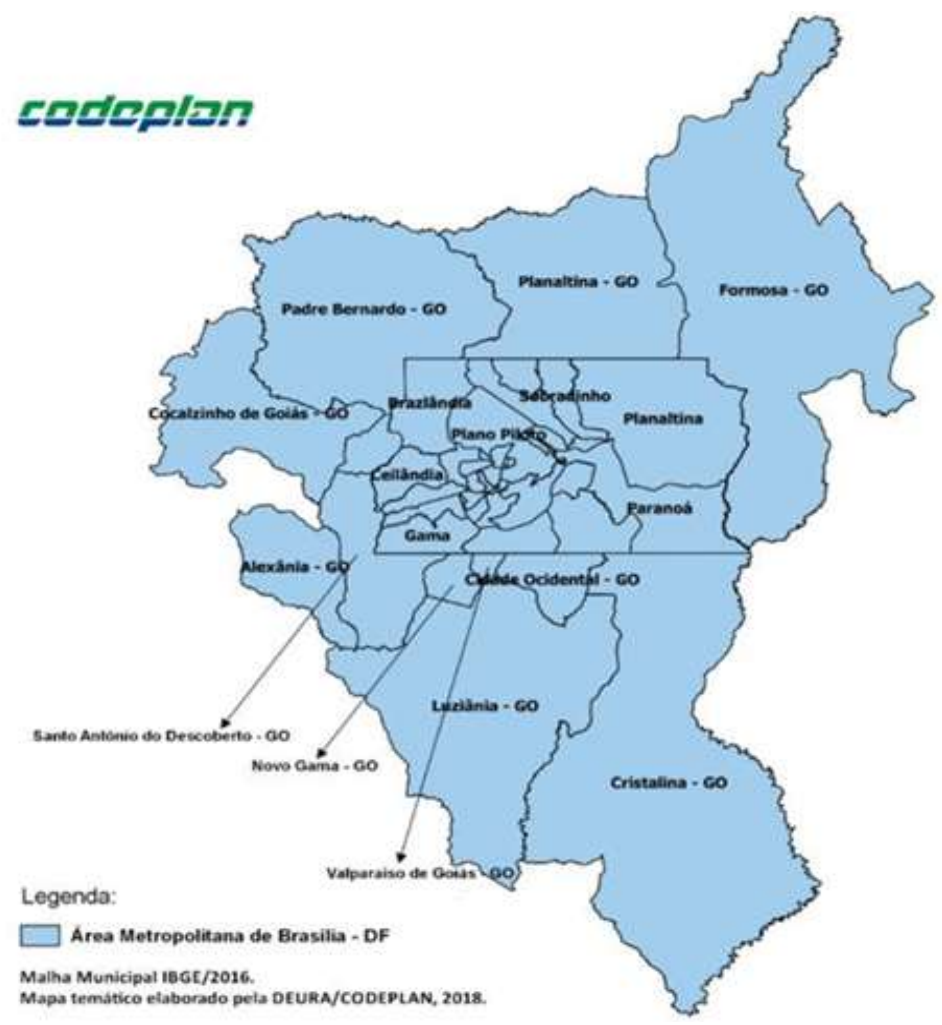

Fonte: CODEPLAN (2014).

\section{RESULTADOS E DISCUSSÃO}

Ao analisar os dados do Índice da População Atendida com Esgotamento Sanitário foi possível perceber uma acentuada variabilidade na AMB. A maior parte dos municípios apresentam um percentual de atendimento com esgotamento sanitário inferior a 60\% (Figura 2). Somente o Distrito Federal apresentou um percentual de atendimento superior a $80 \%$ em todo o período de análise. O ano de 2018 apresentou instabilidade nos dados da maior parte dos municípios.

O Distrito Federal apresentou leve queda no atendimento, o que pode estar associado à acelerada expansão da população. Além disso, os dados relacionados ao DF merecem uma análise mais criteriosa. No atendimento por rede geral de esgotamento sanitário, regiões administrativas distritais como a Fercal e o Sobradinho II apresentam um atendimento entre $20-50 \%$, bem distante do indicador geral para o DF, compreendido entre 80 e $90 \%$. De maneira oposta, existem regiões no DF que apresentam um atendimento superior a 95\%, o que evidencia a elevada heterogeneidade do atendimento como um todo, indicando que nas análises a serem realizadas sobre essa unidade da Federação os dados apresentados retratam valores médios. 

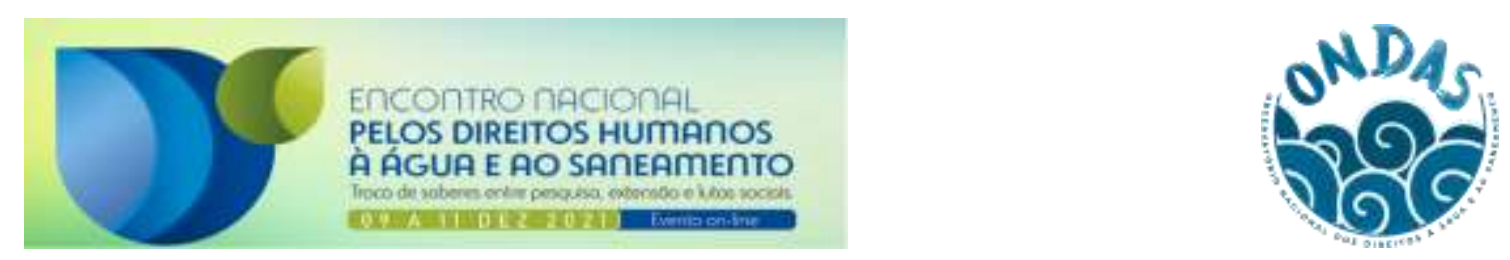

A evolução dos dados do Índice da População Atendida com Esgotamento Sanitário na AMB pode ser visualizada na Figura 3. Os municípios de Alexânia e Cocalzinho de Goiás ainda não dispõem desse tipo de atendimento (dados zerados ou não informados ao SNIS). Existem municípios, como Luziânia/GO, que apresentaram uma evolução muito lenta, sem muitos avanços no período analisado. Alguns municípios apresentaram sinais de melhoria no atendimento, com destaque para Formosa/GO que em 2007 apresentava níveis inferiores a 20\% e em 2018 ultrapassou o valor de 80\% de atendimento.

Figura 2 - Porcentagem da população atendida com esgotamento sanitário na AMB.

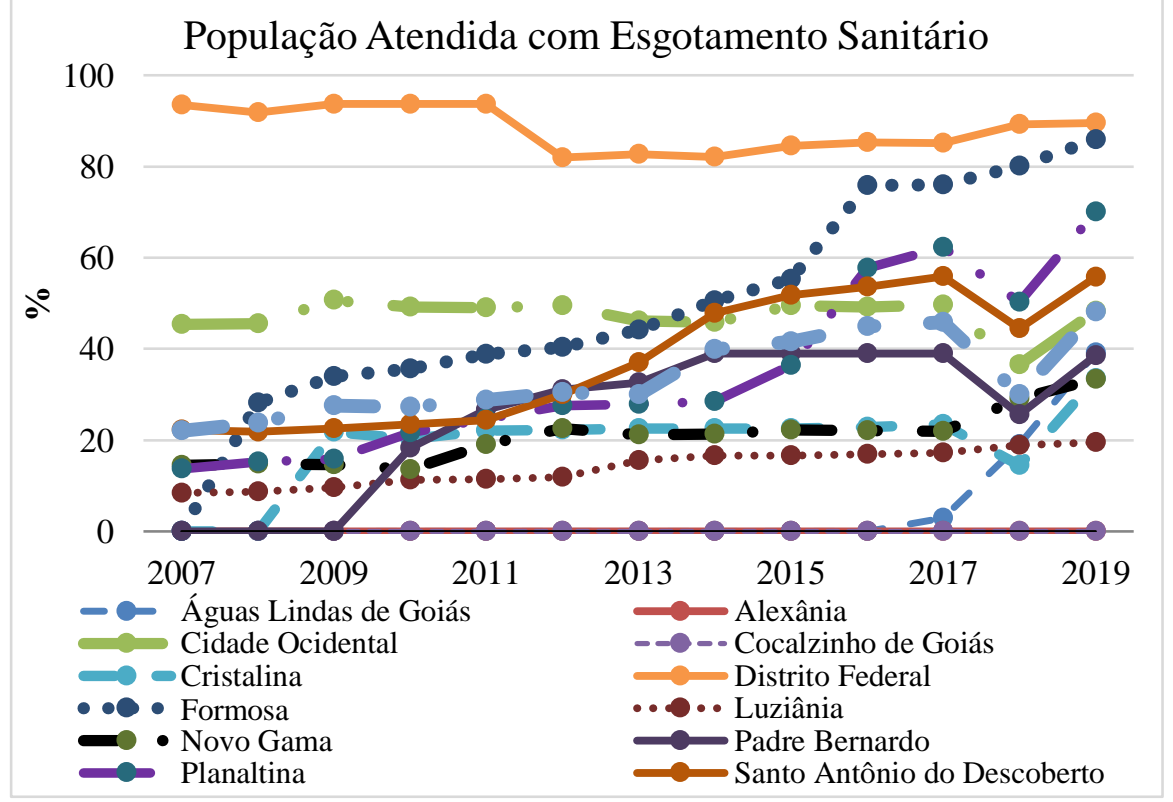

Fonte: SNIS, 2007 a 2019.

Os dados relacionados à ocorrência de Dengue na AMB (Figura 4), no período compreendido entre 2007 e 2019, apresentaram elevada variabilidade. Nos anos inicias da série analisada, 2007 a 2012 , apresentaram valores mais baixos com aumento significativo nos anos de 2013 a 2016; posterior redução e novo aumento em 2019.

No entanto, mesmo com a elevada variabilidade nos dados, em termos espaciais e temporais, observou-se que a maior incidência de Dengue em municípios com as piores taxas de atendimento com esgotamento sanitário.

O Distrito Federal é a única unidade que durante todo o período analisado apresentou os melhores indicadores de saneamento. No entanto, quando comparado a outros municípios, o DF não é a unidade que possui a menor taxa de ocorrência de Dengue. É importante evidenciar que, quando comparado à sua elevada população, os casos de dengue no DF são muito maiores do que nas outras regiões. Seria necessário avaliar os dados do DF de forma individual, analisando os indicadores de dengue e saneamento por Região Administrativa, com intuito de averiguar a correlação entre esses indicadores.

Os casos observados de Leptospirose na AMB (Figura 5), no período compreendido entre 2007 e 2019, foram bem menores do que os casos de Dengue. A diferença entre as incidências tem relação direta com os ciclos de transmissão e a natureza dessas doenças. No entanto, os pequenos aumentos verificados no período também se relacionaram aos municípios com as piores taxas de atendimento com esgotamento sanitário. 
Figura 3 - Mapas temáticos do Índice da População Atendida com Esgotamento Sanitário na AMB.
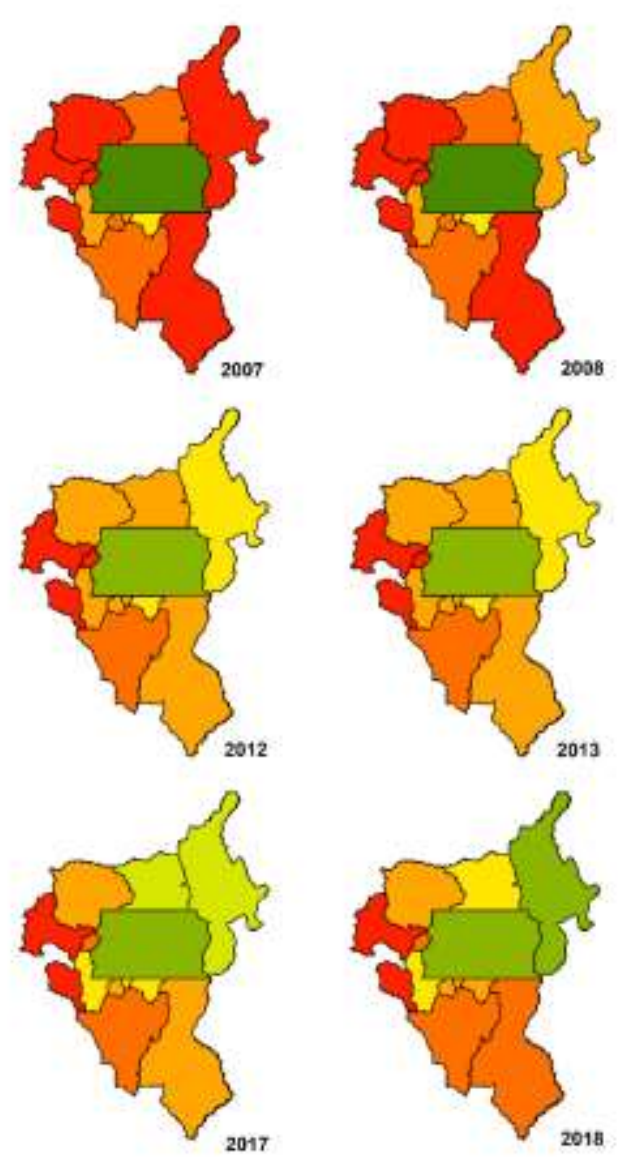

Fonte: SNIS/MDR, 2007 a 2019.
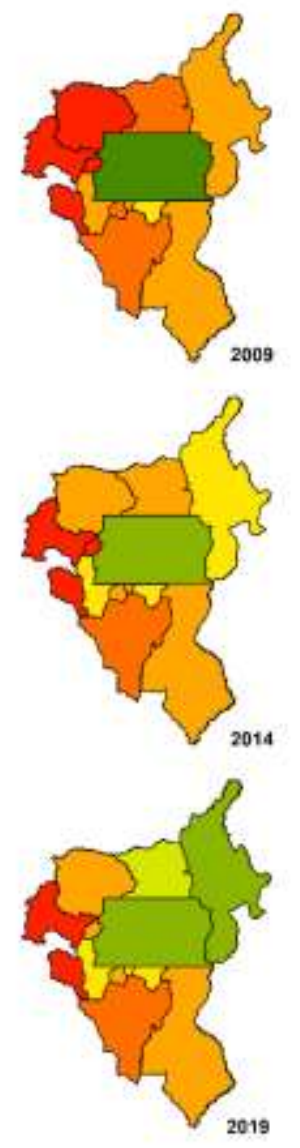
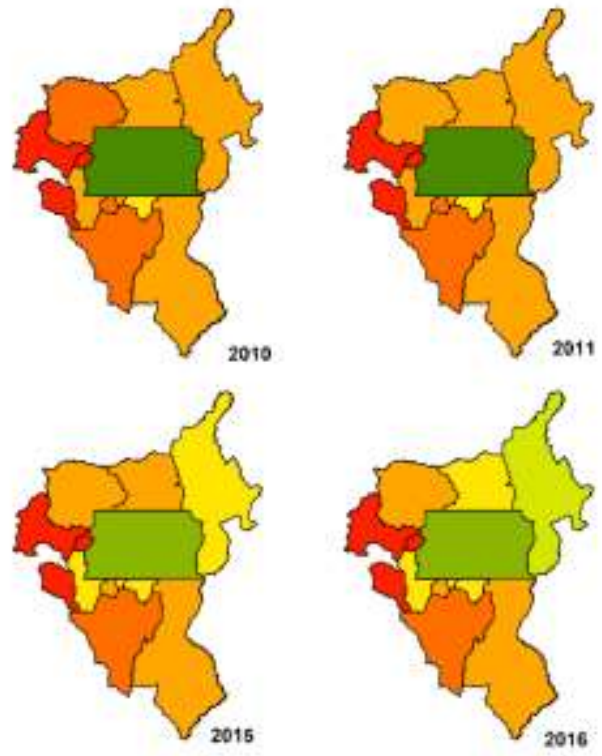

Indice da Populactáo Atendida com Esgotamento Sanitärio

Dons ou Niso incomado

[ $>0 \mathrm{~N}-20 \%$

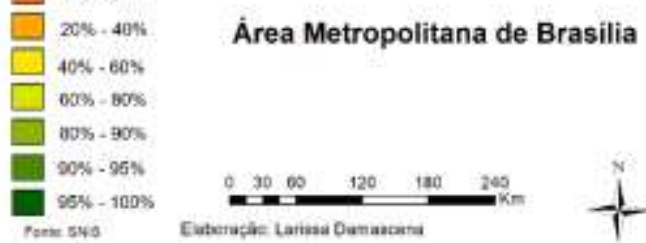

Apesar das análises indicarem uma possibilidade de relação entre o esgotamento sanitário e a ocorrência das doenças analisadas, nos mesmos municípios que apresentarem os piores dados de atendimento de saneamento, verificou-se elevada variabilidade nos dados das doenças, inclusive com valores excessivamente baixos de Dengue e ausência de Leptospirose em determinados anos.

Os resultados reforçam a natureza preliminar da pesquisa e indicam a necessidade de realização de outros estudos para a incorporação de mais dados, inclusive a análise dos dados de outras áreas do saneamento básico.

A associação de dengue e leptospirose com a cobertura de esgotamento sanitário não permitiu verificar se de fato a relação saúde-saneamento se estabelece nessa região. Era esperado que as maiores taxas das doenças estivessem em municípios com baixos índices de atendimento.

\section{CONCLUSÕES}

É importante destacar que a somente a cobertura dos serviços de saneamento não traz resultados no que diz respeito à incidência de doenças. Os hábitos e o uso de maneira responsável desses serviços pelos habitantes devem ser examinados e incorporados nas análises. As bases de dados utilizadas também possuem uma fragilidade em suas informações. A utilização de outras bases de dados poderia contribuir para a pesquisa. Outra grande contribuição para a análise seria a utilização de dados primários, sendo possível verificar a realidade local do saneamento em toda a Área Metropolitana de Brasília. 

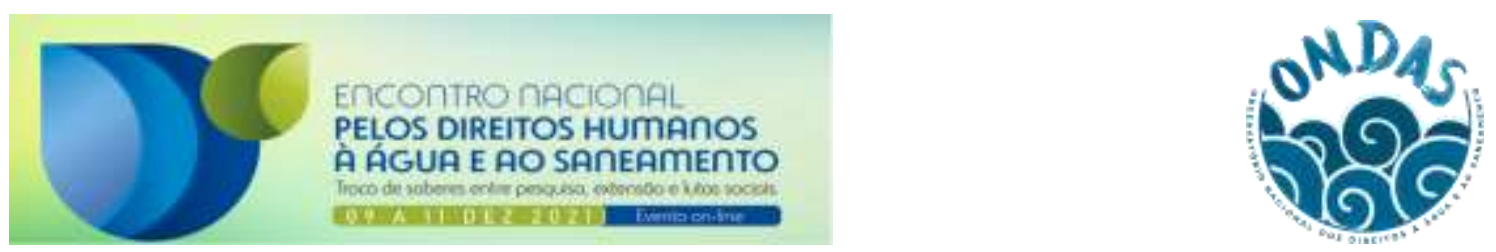

Figura 4 - Mapas temáticos do Índice da População Atendida com Esgotamento Sanitário e Taxa de Ocorrência de Dengue/100.000 habitantes na AMB, nos anos de 2007 a 2019.
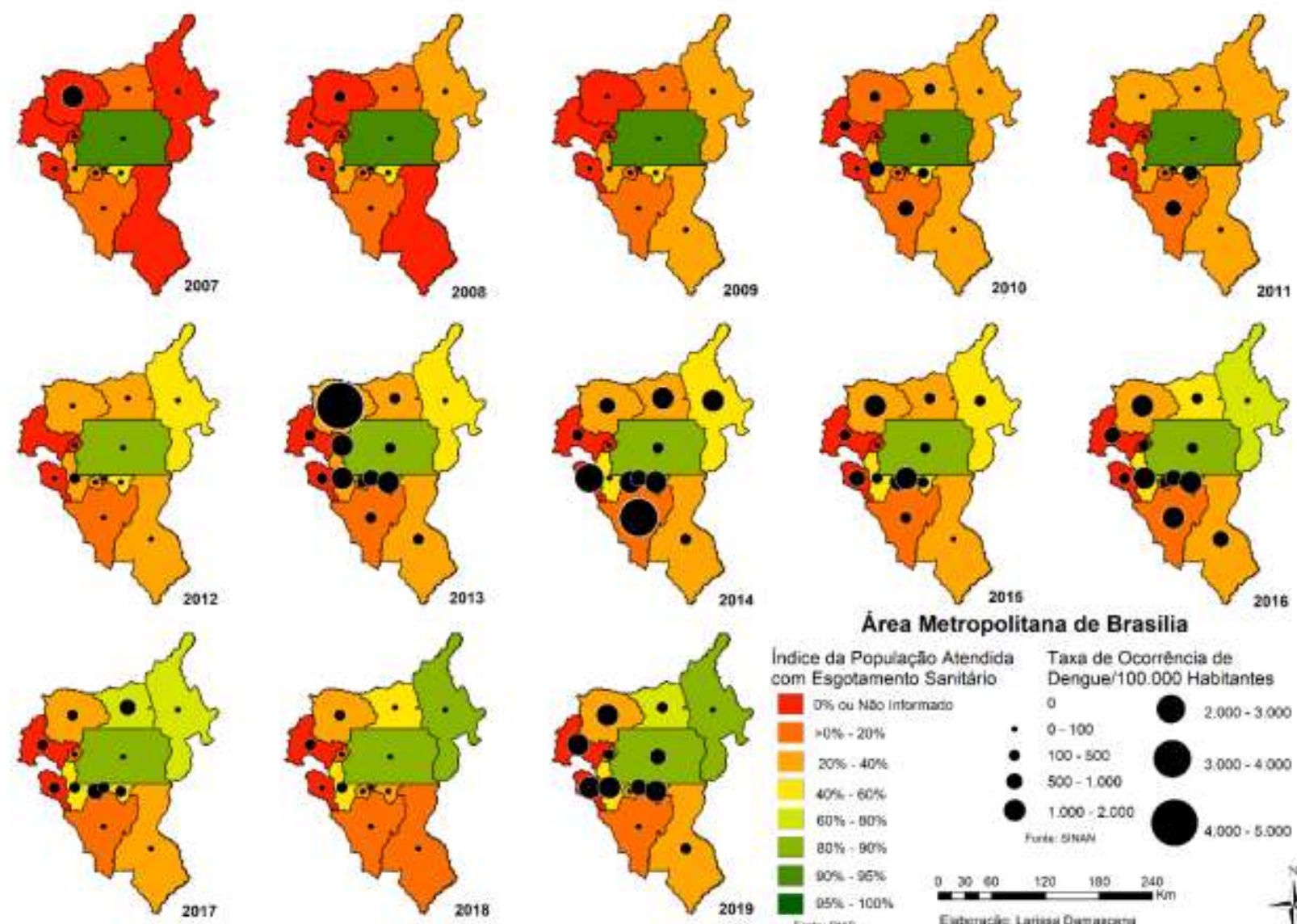

Área Metropolitana de Brasilia

Indice da Populaçio Atendida Taxa de Ocorrệncia de com Esgotamento Sanitário Dengue/100.000 Habitantes

os ou Niso intormatio

[- $>0 \%-20 \%$ 0

- $\quad 0-100$

$2.000-3.000$

$20 \%-40 \%$
$40 \%-60 \%$
$60 \%-00 \%$
$00 \%-90 \%$
$00 \%-96 \%$
$05 \%-100 \%$

- $\quad 0-100$

- $100 \cdot 500$

- $500-1000$

- $1.000: 2.000$

B $3000-4000$

unit: sives

$4.000 \cdot 5.000$
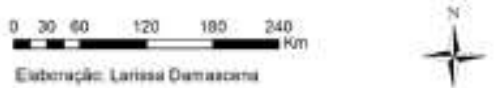

Fonte: SNIS/MDR e SINAN/MS, 2007 a 2019.

Este estudo foi caracterizado como preliminar, com o objetivo de realizar uma análise inicial sobre a associação espacial entre a cobertura de atendimento com esgotamento sanitário e os indicadores de saúde na AMB. Apesar de os resultados deste estudo não permitirem estabelecer a associação direta entre o índice de atendimento e a ocorrência das doenças analisadas, eles mostram que existem muitas diferenças nos indicadores de saneamento na Área e evidencia a necessidade de diminuição das desigualdades na cobertura dos serviços de saneamento básico, visando à melhoria das condições de saúde das populações. 

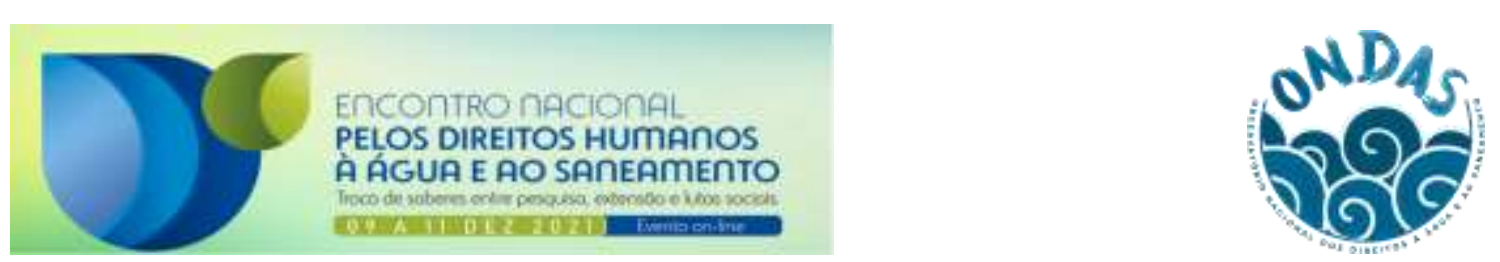

Figura 5 - Mapas temáticos do Índice da População Atendida com Esgotamento Sanitário e Taxa de Ocorrência de Leptospirose/100.000 habitantes na AMB, nos anos de 2007 a 2019.
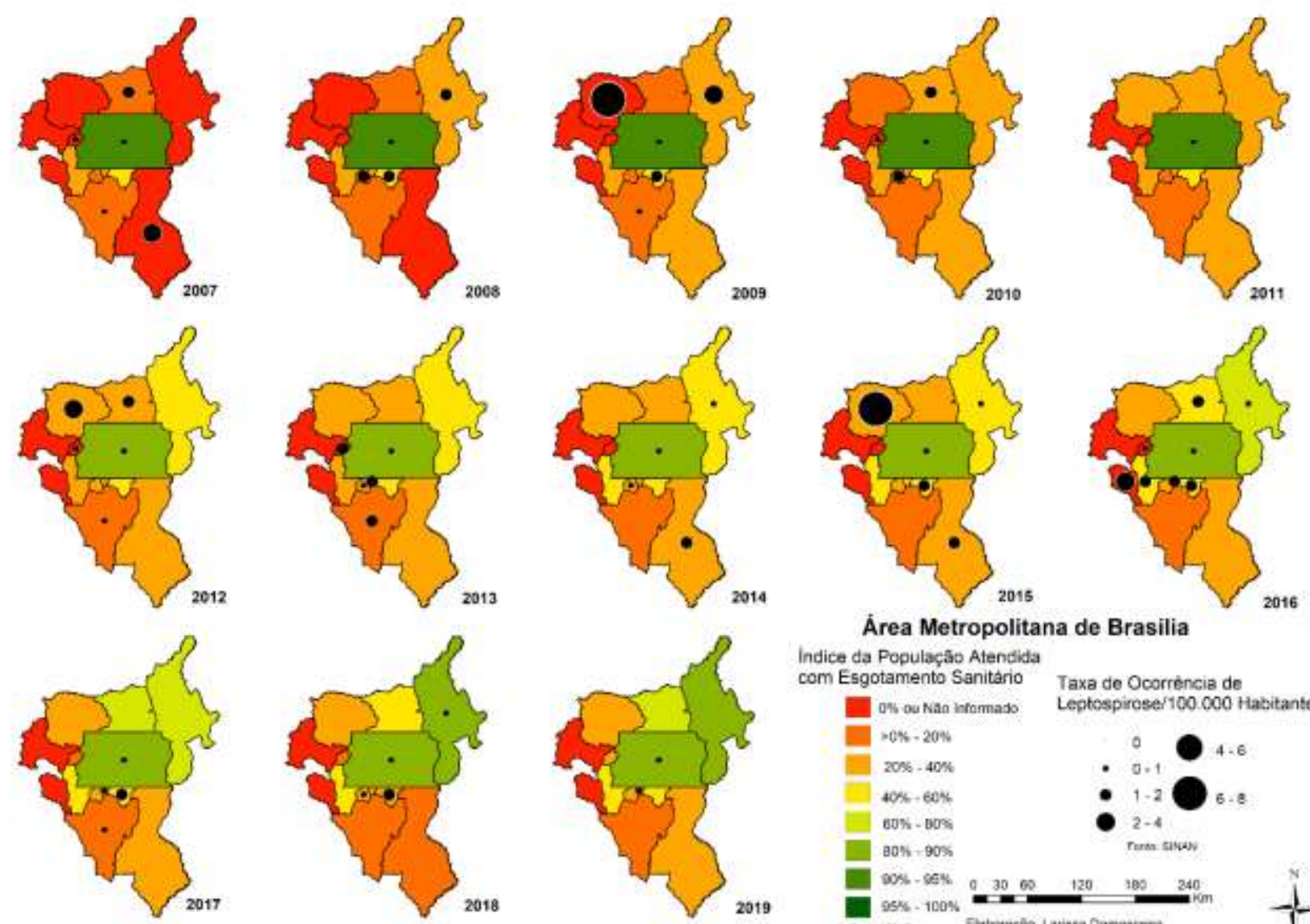

Área Metropolitana de Brasilia

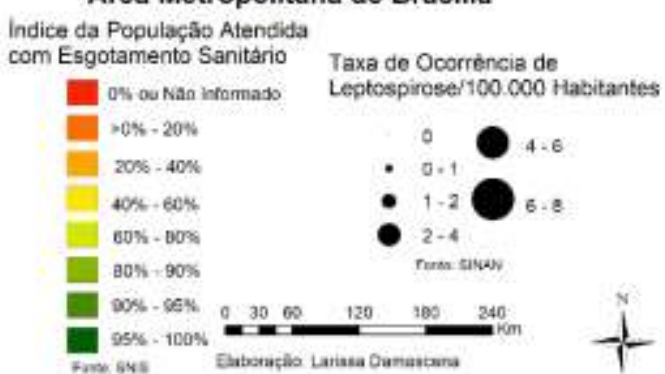

Fonte: SNIS/MDR e SINAN/MS, 2007 a 2019.

\section{REFERÊNCIAS}

CODEPLAN. Companhia de Planejamento do Distrito Federal. Secretaria de Estado do Planejamento e Orçamento do Distrito Federal. Delimitação do Espaço Metropolitano de Brasília (Área Metropolitana de Brasília). Nota Técnica no 01/2014. Brasília. 2014.

DATASUS. Departamento de Informática do Sistema Único de Saúde. Indicadores e Dados Básicos de Saúde - $\quad$ Brasil - $2021 . \quad$ Disponível em: http://www2.datasus.gov.br/DATASUS/index.php?area=02. Acesso em: 20 fev. 2021.

Figueiredo, B; Lacerda, L; Baracho, R. O.; Cordeiro Netto, O. Panorama do saneamento básico na Região Integrada de Desenvolvimento do Distrito Federal e Entorno: Tipificação dos problemas existentes. 2017.

Disponível

em:

https://www.researchgate.net/publication/322204138_panorama_do_saneamento_basico_na_regiao _integrada_de_desenvolvimento_do_distrito_federal_e_entorno_tipificacao_dos_problemas_existe ntes. Acesso em: 20 abr. 2021

ONU. Nações Unidas. General Assembly. A/RES/64/292. The human right to water and sanitation. 2010. Disponível em http://www.un.org/en/ga/search/view_doc.asp?symbol=A/RES/64/292. Acesso em: 20 jun. 2019.

SCHVARSBERG, B. A carroça ao lado do avião: o direito à cidade metropolitana em Brasília. Cad. 
Metrop., São Paulo, v. 19, n. 38, p. 313-334, abr. 2017.

SNIS. Sistema Nacional de Informações sobre Saneamento, 2021. Séries Históricas. Disponível em: <http://www.cidades.gov.br/serieHistorica/>. Acesso em 20 de fev. 2021. 